\title{
Prognostic impact of the expression/phosphorylation of the BH3-only proteins of the BCL-2 family in glioblastoma multiforme
}

\author{
PF Cartron ${ }^{*, 1,2}$, D Loussouarn ${ }^{1,3}$, M Campone $^{4}$, SA Martin ${ }^{1,5}$ and FM Vallette ${ }^{1,2,4}$
}

Apoptosis has a crucial role in anti-cancer treatment. The proteins of the BCL-2 family are core members of the apoptotic program. Thus, we postulated that alterations in the expression of BCL-2 protein family, and in particular in that of the Bcl-2 homology domain 3 (BH3)-only proteins (which can neutralized anti-apoptotic proteins or activate pro-apoptotic proteins) could account for differences in the overall survival (OS) of patients. To test this hypothesis, we analyzed the expression of 15 members of the BCL-2 protein family (Bax, Bak, Bok, Bcl-2, Bcl-xl, Bcl-w, Mcl-1, Bad, Bid, Bim, Bik, Bmf, Hrk, Noxa and Puma) in glioblastoma multiforme (GBM) tumors, the most frequent brain tumor in adults. We found that none of the individual expression of these proteins is associated with a significant variation in OS of the patients. However, when all BH3 proteins were pooled to determine a $\mathrm{BH}^{\text {score }}$, this score was significantly correlated with OS of GBM patients. We also noted that patients with a have high level of phospho-Bad and phospho-Bim displayed a lower OS. Thus, BH3 scoring/profiling could be used as an independent prognostic factor in GBM when globally analyzed.

Cell Death and Disease (2012) 3, e421; doi:10.1038/cddis.2012.150; published online 15 November 2012

Subject Category: Cancer

Malignant gliomas are the most common and deadly brain tumors. Survival for patients with glioblastoma multiforme (GBM), the most aggressive glioma, is individually variable and remains poor although it has improved over the last 5 years. ${ }^{1}$ The major advance in GBM treatment has been following the concomitant use of temozolomide, an alkylating agent, with radiotherapy. ${ }^{2}$ Several publications have focused on the understanding and description of mechanisms by which this regimen limits the growth of these tumors in order to improve the therapeutic impact. In most cases, induction of the cell death program known as apoptosis has been shown to be instrumental in the therapeutic success in GBM. ${ }^{3-5}$

The BCL-2 family of proteins appears to be central in both the initiation and execution of apoptosis, which is composed of three subgroups: the anti-apoptotic proteins, the multi-domain pro-apoptotic proteins and the Bcl-2 homology domain 3 (BH3)-only pro-apoptotic proteins (Figure 1). ${ }^{6}$ The antiapoptotic proteins (i.e., Bcl-2, Bcl-xl, Bcl-w and $\mathrm{Mcl}-1$ ) sequester the pro-apoptotic proteins (i.e., Bax, Bok and Bak) that otherwise, upon activation, permeabilize the outer mitochondrial membrane, enabling the release of cytochrome $c$, promoting the activation of caspases and thereby apoptosis. $^{7,8}$ The BH3-only pro-apoptotic proteins are the first sensors of death signals and trigger apoptosis by inhibiting the anti-apoptotic BCL-2 proteins or by directly activating proapoptotic proteins, Bax and Bak, their $\mathrm{BH} 3$ domains being involved in both mechanisms. ${ }^{9}$ Thus, it is largely assumed that the level of expression of members of the BCL-2 protein family and the interaction between anti- and pro-apoptotic proteins determines life or death in cancer cells and thus the resistance or sensitivity to anti-cancer treatments. Several papers have been devoted to the analyses of the interactions between proand anti-apoptotic proteins, and in particular, between the $\mathrm{BH} 3-$ only proteins and the anti-apoptotic proteins and their putative relationships to the response to anti-cancer treatment. ${ }^{10}$ Indeed, it has been shown that this 'BH3 profiling' can also predict sensitivity to conventional chemotherapeutic agents such as etoposide, vincristine and adriamycin. In addition, expression level of members of the BCL-2 family of proteins appears to be a marker of the therapeutic response and/or of the clinico-pathological characteristic in some cancers. ${ }^{11-13}$

In the present investigation, we have analyzed the expression of several members of the BCL-2 family of proteins (Bax, Bak, Bcl-2, Bcl-xl, Bcl-w, Mcl-1, Bad, Bid, Bim, Hrk, Noxa and Puma) in GBM patients and correlated this expression to a

\footnotetext{
${ }^{1}$ Centre de Recherche en Cancérologie Nantes-Angers, INSERM, U892, Equipe 'Apoptose et progression tumorale', Equipe labellisée Ligue Nationale Contre le Cancer, CRCNA, Nantes, France; ${ }^{2}$ Département de Recherche en Cancérologie, Faculté de Médecine, Université de Nantes, Nantes, France; ${ }^{3}$ Service d'Anatomie Pathologique, HGRL, CHU Nantes-Hopital G et R Laennec, Nantes, France; ${ }^{4}$ Institut de Cancérologie de l'Ouest (ICO), Nantes-Atlantique, Saint Herblain, France and ${ }^{5}$ Service de neurochirurgie, CHU Nantes-Hopital G et R Laennec, Nantes, France

*Corresponding author: PF Cartron, Centre de Recherche en Cancérologie Nantes-Angers, INSERM, U892, Equipe 'Apoptose et progression tumorale', Equipe labellisée Ligue Nationale Contre le Cancer, CRCNA, 8 quai moncousu, Nantes 44007, France. Tel: +33 228080327 ; Fax: + 33 22 808 0204; E-mail: pierrefrancois.cartron@univ-nantes.fr

Keywords: glioma; apoptosis; Bcl-2 proteins; BH3-only proteins.

Abbreviations: $\mathrm{BH} 3, \mathrm{Bcl}-2$ homology domain 3; CPHSRA, Cox proportional hazards survival regression analysis; GBM, glioblatoma multiforme; KPS, Karnofsky Performance Score; OS, overall survival

Received 12.12.11; revised 13.7.12; accepted 22.8.12; Edited by P Salomoni
} 

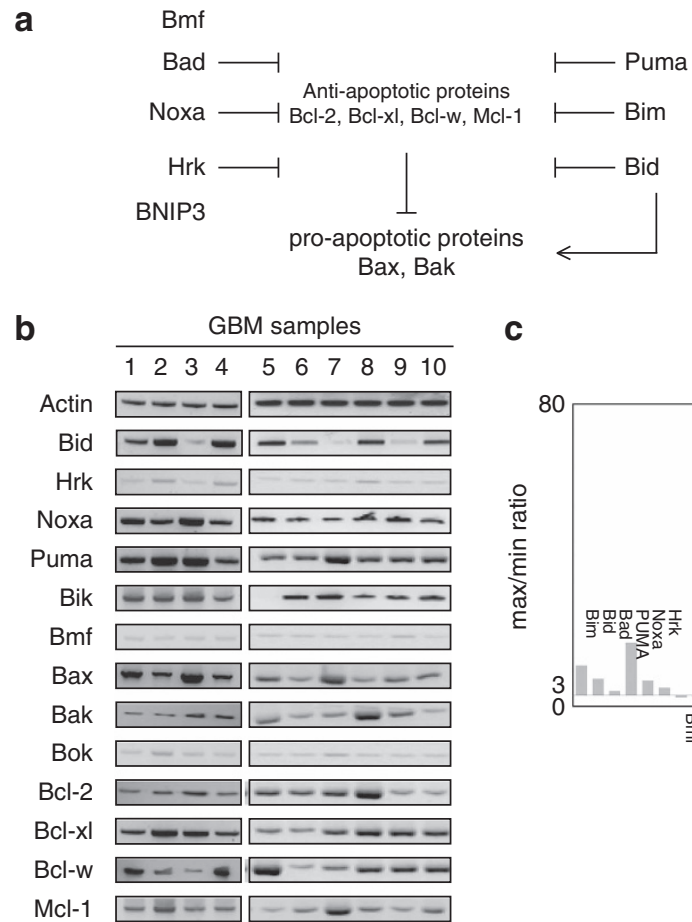

C

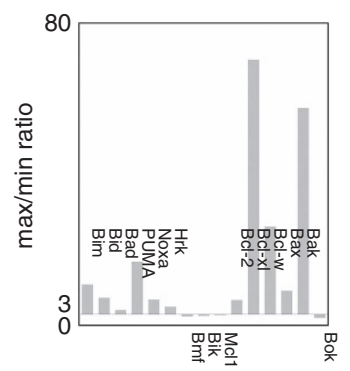

Figure 1 Expression of BCL-2 protein family in GBMs. (a) Model for the apoptotic activity of the BH3-only proteins. (b) Illustration of western blot analyses performed to estimate the expression levels BCL-2 family proteins. Actin was used as an internal control. Western blot assays were performed from $50 \mathrm{mg}$ of protein extract and with the indicated panel of antibodies directed against BCL-2 protein family. (c) Graph representing the ratio between the maximal level of expression $(\max )$ of the considered protein and its minimal level of expression $(\min )$.

relevant gain of overall survival (OS) in patients. Posttranslational modification of the function of BCL-2 family of proteins, and in particular, the pro-apoptotic function of the Bad and Bim, both pro-apoptotic proteins, were also investigated by determining the phosphorylation of these proteins in conjunction with a variation in OS in GBM patients. $^{14}$

\section{Results}

Focus on certain members of the BCL-2 protein family. Initially, we monitored the expression of 13 members of the BCL-2 protein family (Bax, Bak, Bok, Bcl-2, Bcl-xl, Bcl-w, Mcl-1, Bad, Bid, Bik, Bim, Bmf, Hrk, Noxa and Puma) in 10 GBM using western blotting (WB) and ELISAs (Figure 1b). As illustrated in Figure 1b, the expression of Mcl-1, Bmf, Bik and Bok was homogeneous, whereas the expression level of the other BCL-2 proteins was heterogeneous. In addition, by analyzing the $10 \mathrm{GBM}$, we observed that the ratio between the maximal and minimal value of expression was inferior to three for the $\mathrm{Mcl}-1, \mathrm{Bmf}, \mathrm{Bik}$ and Bok proteins and was superior to three for the other members of the BCL-2 protein family (Figure 1c).

As a consequence, we decided to perform in-depth analyses of all members of BCL-2 protein family harboring a heterogeneous expression, that is, Bax, Bak, Bcl-2, Bcl-xl, Bcl-w, Bad, Bid, Bim, Hrk, Noxa and Puma proteins.

The population analyzed in this study is representative of GBM patients. The study population consisted of 31 GBM patients for which we obtained a well-documented medical history and molecular characteristics according to French laws and recommendations of the French National Committee of Ethics. All these patients had received the same standard treatment following a complete surgical resection (that is, Temozolomide plus irradiation). We were also able to accurately determine and compare the time to OS in this population. The patients had a median OS of 10.5 months, a male/female proportion of $20 / 11$, a KPS with a median of 80 (Table 1). Of note, the CPHSRA showed no prognostic relevance of the latter clinical parameters (Table 1). We complemented the characterization of the population by analyzing two GBM-associated basic molecular parameters: MGMT methylation status and p53 mutation. In this cohort of 31 patients, 29\% harbored a methylated MGMT gene, which conferred a superior OS as compared with patients with an unmethylated MGMT gene $(P=0.0134)$. On the other hand, the p53 mutation was detected in $26 \%$ of the patients and was not associated with a prognostic relevance in OS $(P=0.6462)$ (Table 1). In addition, no correlation was observed between p53 status and the degree of methylation of the MGMT gene $(P=0.2454)$.

These results were in agreement with current data and showed that our population could be considered representative of GBM patients. ${ }^{1}$

Individual expression of key members of the BCL-2 protein family is not prognostic of OS. In each GBM samples, we analyzed the expression of key members of the $\mathrm{BCL}-2$ protein family in order to determine whether or not this influenced the response of patients to the irradiation plus temozolamide treatment. ${ }^{6}$ According to previous results, the 11 members of the BCL-2 protein family analyzed were $\mathrm{Bcl}-2$, Bcl-xl, Bcl-w (the major three anti-apoptotic proteins in GBM), Bax and Bak (two multi-domain pro-apoptotic proteins) and Bad, Bid, Bim, Hrk, Noxa and PUMA, six major BH3-only pro-apoptotic proteins. Western blot or ELISA experiments were performed to estimate the expression level of these proteins (Figure 2).

First, we observed that the expression of BCL-2 protein family was very heterogeneous among GBM patients (Figure 2 and Table 1). Indeed, this idea can be illustrated by the fact that Noxa expression varied between $(0.24 ; 6.54)$, that is, that the maximal value of Noxa expression is equal to 27-fold its minimal value. Similar observations were also made for $\mathrm{Bcl}-\mathrm{xl}$ (maximal value of expression is equal to 70 -fold the minimal value) and for Bak (maximal value is equal to 58 -fold the minimal value).

Second, we searched for potential correlations between the expression of the different members of the BCL-2 protein family. We found significant correlations between the expression of Bcl-2 and Bcl-w ( $r=0.375$ and $P=0.0376)$, Bcl-2 and Bax $(r=0.614$ and $P=0.0002)$, Bax and Bcl-w $(r=0.362$ and $P=0.0454)$ and Bak and $\operatorname{Bim}(r=0.377$ and $P=0.0366)$ 
Table 1 Characteristics of patients and analyses of overall survival

\begin{tabular}{|c|c|c|c|c|}
\hline & $n$ & Median & $($ Min, $\max )$ & CPHSRA (P-value) \\
\hline Overall survival (months) & 31 & 10.5 & $(3.2,36.2)$ & - \\
\hline Age (years) & 31 & 60.2 & $(48.2,67.4)$ & 0.5159 \\
\hline $\operatorname{Sex}(M / F)$ & $24 / 11$ & - & - & 0.7377 \\
\hline $\mathrm{KPS}<90$ versus $\mathrm{KPS}=90$ & 31 & 80 & $(70,90)$ & 0.3743 \\
\hline $\mathrm{p} 53^{\text {wt }}$ versus $\mathrm{p} 53^{\text {mut }}$ & $23 / 8$ & - & - & 0.6462 \\
\hline MGMT $^{\mathrm{U}}$ versus MGMT ${ }^{\mathrm{M}}$ & $22 / 9$ & - & - & 0.0134 \\
\hline $\mathrm{Bad}^{\text {low }}$ versus Bad $\mathrm{Bdigh}^{\text {hin }}$ & $16 / 15$ & 3.25 & $(1.06,5.26)$ & 0.9192 \\
\hline Bid $^{\text {low }}$ versus Bid ${ }^{\text {high }}$ & $16 / 15$ & 1.65 & $(0.62,6.24)$ & 0.5872 \\
\hline Bim $^{\text {low }}$ versus Bim ${ }^{\text {high }}$ & $16 / 15$ & 1.35 & $(0.57,6.17)$ & 0.1269 \\
\hline Hrk $^{\text {low }}$ versus Hrk ${ }^{\text {high }}$ & $16 / 15$ & 4.01 & $(0.51,7.45)$ & 0.1257 \\
\hline Noxalow versus Noxa high & $15 / 16$ & 2.35 & $(0.24,6.54)$ & 0.1416 \\
\hline Pumal $^{\text {low }}$ versus Puma high & $15 / 16$ & 2.51 & $(0.44,7.45)$ & 0.1983 \\
\hline $\mathrm{Bcl}-2^{\text {low }}$ versus $\mathrm{Bcl}-2^{\text {high }}$ & $16 / 15$ & 4.25 & $(0.98,8.75)$ & 0.4406 \\
\hline $\mathrm{Bcl}-\mathrm{xl}^{\text {low }}$ versus $\mathrm{Bcl}-\left.\mathrm{xl}\right|^{\text {high }}$ & $16 / 15$ & 5.21 & $(0.12,8.45)$ & 0.7567 \\
\hline $\mathrm{Bcl}^{\text {low }}{ }^{\text {low }}$ versus $\mathrm{Bcl}-\mathrm{w}^{\text {high }}$ & $17 / 14$ & 3.21 & $(0.12,7.68)$ & 0.2836 \\
\hline Bax ${ }^{\text {low }}$ versus Bax ${ }^{\text {high }}$ & $16 / 15$ & 3.03 & $(0.87,8.98)$ & 0.5340 \\
\hline Bak $^{\text {low }}$ versus Bak ${ }^{\text {high }}$ & $17 / 14$ & 2.24 & $(0.14,8.06)$ & 0.2773 \\
\hline $\mathrm{pBad}^{\mathrm{S136-low}}$ versus $\mathrm{pBad}{ }^{\text {S136-high }}$ & $16 / 15$ & 2.05 & $(0.21,6.21)$ & 0.6129 \\
\hline pBim $^{\text {s69-low }}$ versus pBim ${ }^{\text {S69-high }}$ & $16 / 15$ & 1.36 & $(0.14,6.24)$ & 0.3498 \\
\hline
\end{tabular}

Abbreviations: CPHSRA, Cox proportional hazards survival regression analysis; F, female; KPS, Karnofsky Performance Score; M, male; MGMTM, methylated MGMT; MGMT ${ }^{\mathrm{U}}$, unmethylated MGMT; p53 ${ }^{\text {mut }}$, p53 mutations; $553^{\text {wt }}$, wild-type p53.

Overall survival data were analyzed using CPHSRA and significance $(P)$ was reported in the table for each analyzed parameter (age, sex, KPS, presence of wild-type p53 (p53 ${ }^{\text {wt }}$ ) or p53 mutations (p53 ${ }^{\mathrm{mut}}$ ), expression level of the considered member of the BCL-2 protein family.

(Figure 3a-d). A significant inverse correlation was observed between the expression level of Bad and Noxa $(r=-0.498$ and $P=0.044$ ) (Figure 3e).

Third, we determined if correlations between the expression of the different members of the BCL-2 protein family and the GBM-associated basic molecular parameters included in our study existed. Our analysis revealed that the expression level of Bak was correlated with the presence of p53 mutation ( $r=0.464$ and $P=0.0086$ ) (Figure 4a), while the presence of a methylated MGMT gene correlated with the expression level of Bak and Bim (Figure 4b) $(r=0.378$ and $P=0.0360$ and $r=0.485$ and $P=0.0057$, respectively).

Fourth, we analyzed whether the expression level of these members of the BCL-2 protein family was associated with of a prognostic relevance of OS. For this, patients were divided into two groups according to the expression level of the 11 members of the BCL-2 protein family: one with low expression levels of the protein (i.e., lower or equal to the median) and a second group included GBM patients with high levels of expression (i.e., higher than the median). CPHSRA revealed that none of the protein expression levels was associated with of a prognostic relevance in OS (Table 1).

These results suggest that there was no simple correlation between the individual expression of our selected members of the BCL-2 protein family and patient OS.

A 'BH3 score' correlates with OS in GBM patients. As the individual expression of members of the BCL-2 protein family was not an independent prognostic factor in OS, we considered the effect of a 'pooled expression' of antiapoptotic proteins $\left(A A^{\text {score }}\right)$, of multi-domain pro-apoptotic proteins (mPA ${ }^{\text {score }}$ ) and of $\mathrm{BH}$-only pro-apoptotic proteins $\left(\mathrm{BH} 3^{\text {score }}\right)$. For the calculation of each score, 1 point was attributed when a member of the BCL-2 protein family harbored a high expression level (higher than the median),
0 point was attributed when the member of $B C L-2$ protein family harbored a low expression level (lower or equal to the median).

An initial analysis revealed that only the $\mathrm{BH} 3^{\text {score }}$ significantly correlated with the OS (AA ${ }^{\text {score }}$ versus OS: $P=0.9536$; $\mathrm{mPA}^{\text {score }}$ versus OS: $P=0.6313$; $\mathrm{BH}^{\text {score }}$ versus OS: $P=0.0180$ ) (Figure 5a). In other terms, our analyses indicated that a high expression of $\mathrm{BH} 3-o n l y$ proteins, correlated to a longer OS.

On the basis of this observation, we refined our $\mathrm{BH}^{\text {score }}$. For this purpose, we considered three different $\mathrm{BH} 3^{\text {score }}$. The first consisted of a 'BH3 ${ }^{\text {score }}<3^{\prime}$ group that included GBM patients with high expression in less than three BH3-only proteins. The second consisted of a ' $\mathrm{BH} 3^{\text {score }}=3$ group' that included GBM patients presenting three high expression $\mathrm{BH} 3-$ only proteins (higher than the median value) and the third, a ' $\mathrm{BH}^{\text {score }}>3^{\prime}$ group that included patients with more than three highly expressed $\mathrm{BH} 3$-only proteins.

CPHSRA showed that the OS of patients was increased only in the latter group as OS in the ' $\mathrm{BH} 3^{\text {score }}>3^{\prime}$ group was significantly more important than that of the ' $\mathrm{BH} 3^{\text {score }}<3^{\prime}$ group $(P=0.0132)$ (Figure $5 b)$. In addition and more importantly, we noted that this was independent of the type of BH3-only protein.

High level of $\mathrm{pBad}^{\mathrm{S136}}$ and $\mathrm{pBim}^{\mathrm{S} 69}$ is a hallmark associated with poor survival of GBM patients. Numerous reports in the literature state that the phosphorylation of BH3-only proteins can act as a modulator of the proapoptotic function of these proteins, as such we assessed the level of $\mathrm{pBad}^{\mathrm{S136}}$ and $\mathrm{pBim}^{\mathrm{S} 69}$ in our collection of GBM. ${ }^{15,16}$ Results obtained from ELISA firstly indicated a high heterogeneity in the expression of $\mathrm{pBad}^{\mathrm{S} 136}$ and pBim $^{\text {S69 }}$ as a ratio of 30 and 45 were observed between the maximal and the minimal value of the $\mathrm{pBad}^{\mathrm{S136}}$ and 


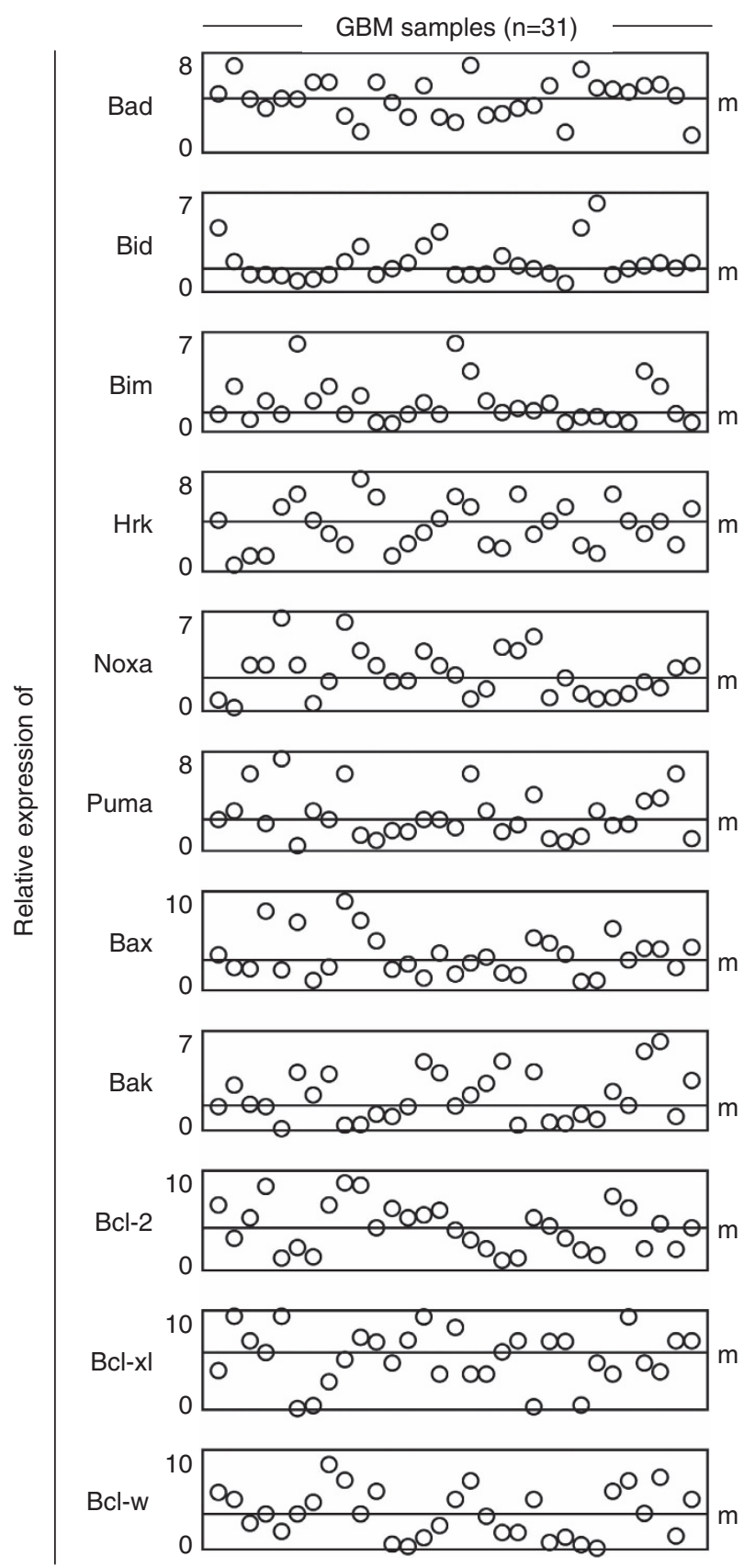

Figure 2 Expression of considered BCL-2 protein family in GBMs. Graphs illustrate the relative expression of each considered member of the BCL-2 protein family in each tumors included in our study (1 open circle $=1 \mathrm{GBM}$ ). M: median. The relative expression of each considered member of the BCL-2 protein family was estimated by western blot or ELISA analyses such as described above.

pBim ${ }^{569}$ expression (Table 1). Second, we noted a correlation between the presence of $\mathrm{pBad}^{\mathrm{S} 136}$ and $\mathrm{pBim}^{\mathrm{S} 69}$ suggesting that this phosphorylation could be because of the same kinase (Figure 6a). Third, CPHSRA revealed that the level of $\mathrm{pBad}^{\mathrm{S136}}$ and $\mathrm{pBim}^{\mathrm{S69}}$ was not associated with a difference in OS between patient harboring low (lower or equal to the median) or high (higher to the median) level of $\mathrm{pBad}^{\mathrm{S} 136}$ and low (lower or equal to the median) or high (higher to the median) level of $\mathrm{pBim}^{\mathrm{S} 69}$ (Figures $6 \mathrm{~b}$ and $\mathrm{c}$ ). Finally, CPHSRA indicated that OS of GBM patients with a high level of $\mathrm{pBad}^{\mathrm{S136}}$ and $\mathrm{pBim}^{\mathrm{S} 69}$ were inferior to $\mathrm{OS}$ in patients without this double hallmark (Figure $6 \mathrm{~d}$ ).

Presence of $\mathrm{pBad}^{\mathrm{S136}}$ and $\mathrm{pBim}^{\mathrm{S} 69}$ in GBM and kinase activities. Finally, we considered whether the level of $\mathrm{pBad}^{\mathrm{S136}}$ and $\mathrm{pBim}{ }^{\mathrm{S69}}$ was correlated with the level of the Akt and Erk activities as these kinases are responsible for the phosphorylation of Bad and Bim at the S136 and S69 residues, respectively. ${ }^{17,18}$ For this purpose, we determined Erk activity by using the MAP Kinase/Erk Immunoprecipitation Kinase Assay Kit, non-radioactive (Milipore, France) and the Akt activity by using the AKT In-Cell ELISA Kit (ThermoScience, Villebon Sur Yvette, France). In addition, we also estimated the level of kinase activity of the EGF receptor (EGFr), by using the HTScan EGF Receptor Kinase Assay Kit (Ozyme/cell signaling, France) as EGFr is overactivated in GBM and able to promote the Akt and the Erk activation. The correlation between these values was then determined by using Pearson's correlation test (Figure 7). Our data indicated that the Akt activity level correlated with the level of $\mathrm{pBad}^{\mathrm{S} 136}$ and with the level of EGFr activities, but not with the level of $\mathrm{pBim}^{\mathrm{S} 69}$, while the Erk activity level correlated with the level of pBim ${ }^{\mathrm{s} 69}$ and with the level of EGFr activities but not with the level of level of $\mathrm{pBad}^{\mathrm{S136}}$.

\section{Discussion}

Our research is focused on the molecular mechanism(s) of apoptosis evasion in GBM as an inhibition of the cell death program in these tumors is an important feature in the resistance to treatment. ${ }^{19}$ In the present study, we have studied the prognostic significance of the expression of 11 members of the BCL-2 protein family in GBM, namely Bcl-2, Bcl-xl, Bcl-w, Bax, Bak, Bad, Bid Bim, Hrk, Noxa and Puma. We found no correlation between the level of expression of any of the aforementioned proteins and OS. However, when the expression of $\mathrm{BH} 3-$ only proteins was pooled to define a $\mathrm{BH}^{\text {score }}$, this score significantly correlated with OS. Indeed this $\mathrm{BH}^{\text {score }}$ could be used as an independent prognostic factor for GBM patients. GBMs with a high expression of two BH3-only proteins (i.e., Bad, Bid, Bim, Hrk, Noxa and Puma) presented a low OS (median: 9.45 months), while tumors exhibiting a high expression of at least four of these BH3-only proteins presented a high OS (median: 14.55 months). Thus, quantification of the expression level of six $\mathrm{BH}$-only proteins could be considered as a biomarker associated with a significant prognosis in terms of OS.

By performing ELISA and WB, we focused on the quantitative level of the expression of the $B C L-2$ protein family at the expense of a study of the location and number of positive cells for the expression of these proteins (data provide by immunohostochemical (IHC) experiments). However, and taking into consideration the relative sensitivity and specificity of these methods, we and others have already shown that the results of studies of ELISA, WB, IHC could be superimposable. 20,21

This observation is one of the first using such a large panel of $\mathrm{BH} 3-$ only proteins. There have been numerous literature 

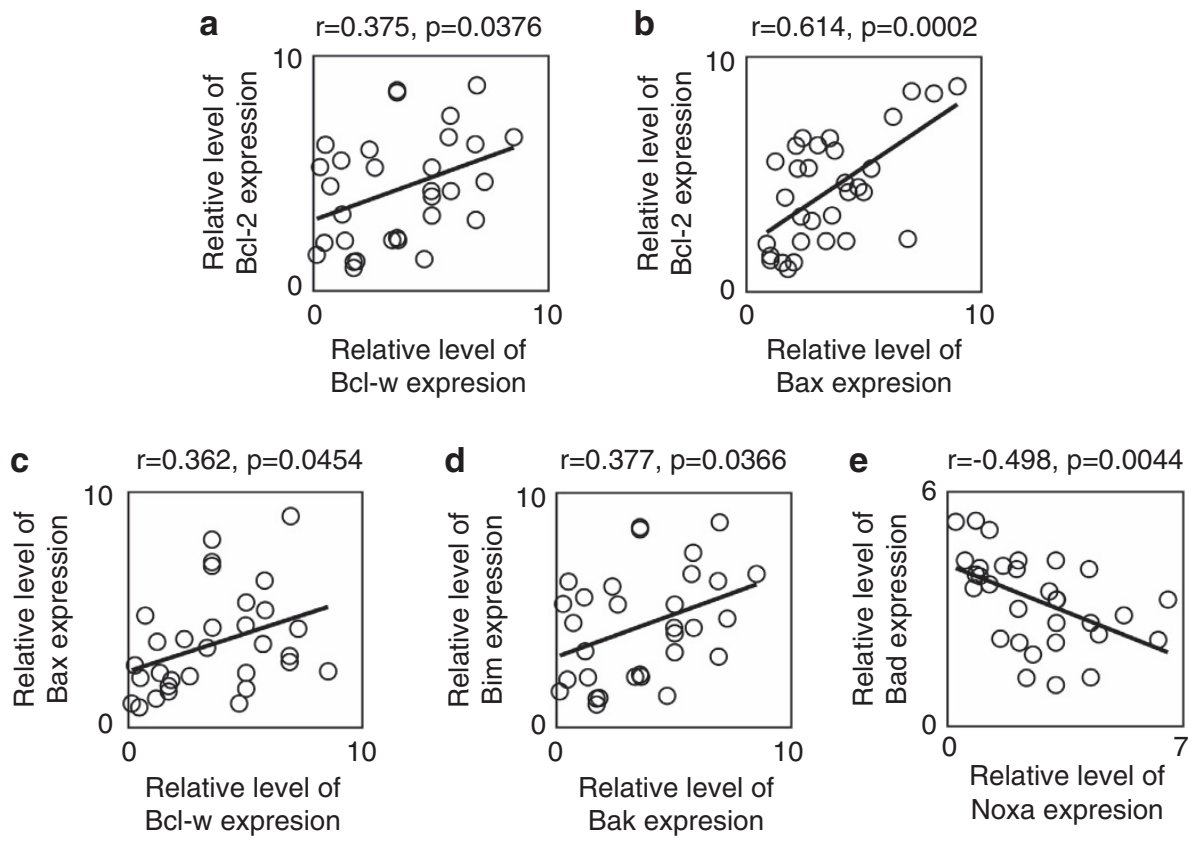

Figure 3 Analyses of correlation between the expression levels of two members of the BCL-2 protein family. Each circle represents one GBM tumor. (a) Correlation between the levels of Bcl-2 and Bcl-w $(P=0.0376$, Pearson's test coefficient $r=0.375)$. (b) Correlation between the levels of Bcl-2 and Bax $(P=0.0002$, Pearson's test coefficient $r=0.614)$. (c) Correlation between the levels of bax and Bcl-w $(P=0.0454$, Pearson's test coefficient $r=0.362)$. (d) Correlation between the levels of Bim and Bak $(P=0.0366$, Pearson's test coefficient $r=0.377)$. (e) Inverse correlation between the levels of Bad and Noxa $(P=0.0044$, Pearson's test coefficient $r=-0.498)$.
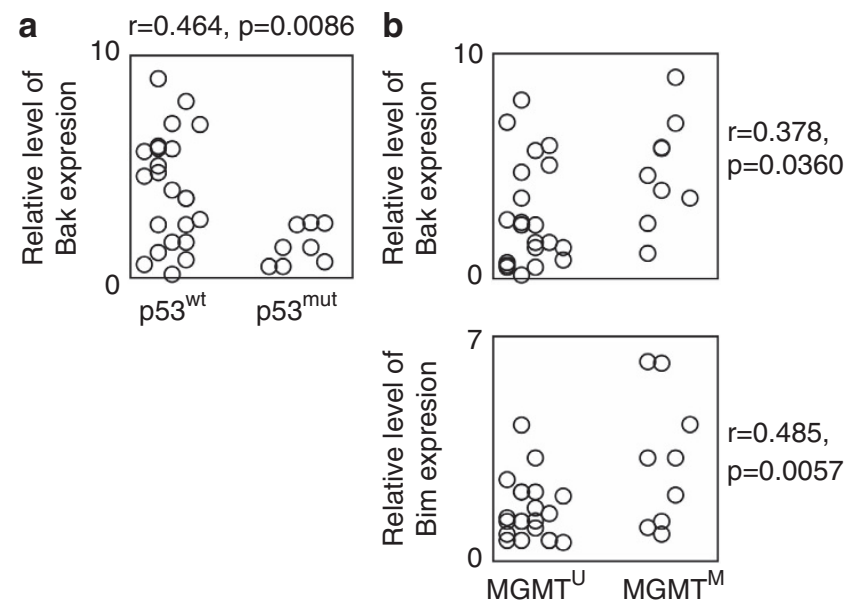

Figure 4 Analysis of the correlation between the expression level of a member of the BCL-2 protein family and the p53 status (wild-type (wt) or mutated (mut)) or the degree of MGMT gene methylation (MGMT: unmethylated MGMT gene or MGMT $^{\mathrm{M}}$ : methylated MGMT gene). Each circle represents one GBM tumor. (a) Correlation between the levels of Bak and the p53 status (wild-type or mutated) $(P=0.0086$, Pearson's test coefficient $r=0.464)$. (b) Correlation between the levels of Bak and the MGMT status (unmethylated or methylated) $(P=0.0360$, Pearson's test coefficient $r=0.378$ ) and correlation between the levels of Bim and the MGMT status (unmethylated or methylated) $(P=0.0057$, Pearson's test coefficient $r=0.485$ ).

reports about the expression of BH3-only protein(s) and OS. ${ }^{16-18}$ The expression level of a single $\mathrm{BH}$-only protein was shown to significantly correlated with survival (such as Bim for melanoma patients). ${ }^{22}$ Other groups have reported that $\mathrm{BH} 3-$ only proteins Bim and Puma can provide prognostic information for stage II and III colon cancer patients receiving 5-fluorouracil-based adjuvant chemotherapy while Bad and Bid expression predicted survival in stage II and III colon cancers. $^{23,24}$

Other groups have reported that $\mathrm{BH}$-only proteins Bim and Puma can provide prognostic information for stage II and III colon cancer patients receiving 5-fluorouracil-based adjuvant chemotherapy, while Bad and Bid expression predicted survival in stage II and III colon cancers. ${ }^{16}$

The co-existence of $\mathrm{pBad}^{\mathrm{S} 136}$ and $\mathrm{pBim}^{\mathrm{S} 69}$ expression, despite the fact that distinct kinases promote the $\mathrm{pBad}^{\mathrm{S136}}$ and pBim $^{\mathrm{S} 69}$ phosphorylation can be explain by the fact that kinases governing these phosphorylations, that is, Akt and Erk can be activated by the same signaling pathway such as the one of EGR/EGFr pathway. Our data support this idea as we observed that the kinase activity of Akt and Erk was correlated with the kinase activity of EGFr.

In addition, our study also shows the existence of a correlation between the expression levels of several members of the BCL-2 protein family. This study confirms the existence of a parallel increase between the expression of $\mathrm{Bcl}-2$ and $\mathrm{Bax}$ in GBM tumors, as previously described by Martin et al. ${ }^{21}$ Here, we complement this point by noting that the expression level of Bcl-w also correlates with the expression level of Bax and $\mathrm{Bcl}-2$. Taken together these data suggest that $\mathrm{Bax}, \mathrm{Bcl}-2$ and $\mathrm{Bcl}-\mathrm{w}$ can be co-regulated by similar mechanisms in GBM. This might be related to the fact that methylation of the $b c l-2$ and $b c l-w$ genes occurs frequently in the same GBM tumor. ${ }^{25}$ Strikingly, our data suggest the existence of a correlation between the expression of Bak and Bim and the presence of an inverse correlation between the expression levels of Bad and Noxa. The co-regulation between Bim and 
a

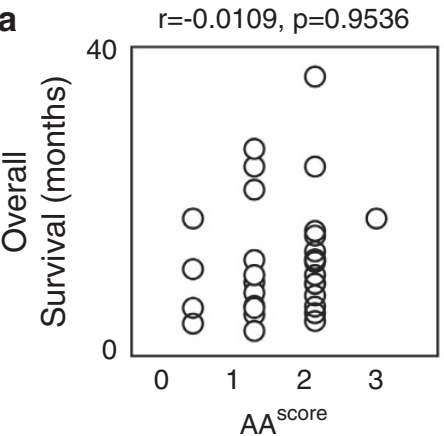

b

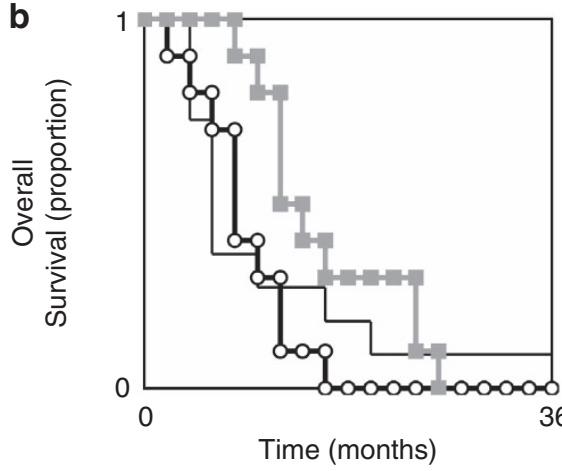

-O- $\mathrm{BH}^{\text {score }}<3, \mathrm{n}=10, \mathrm{~m}=9.45$ months

- $\mathrm{BH}^{\text {score }}=3, \mathrm{n}=11, \mathrm{~m}=6.5$ months

- $\mathrm{BH}^{\text {score }}>3, n=10, m=14.55$ months
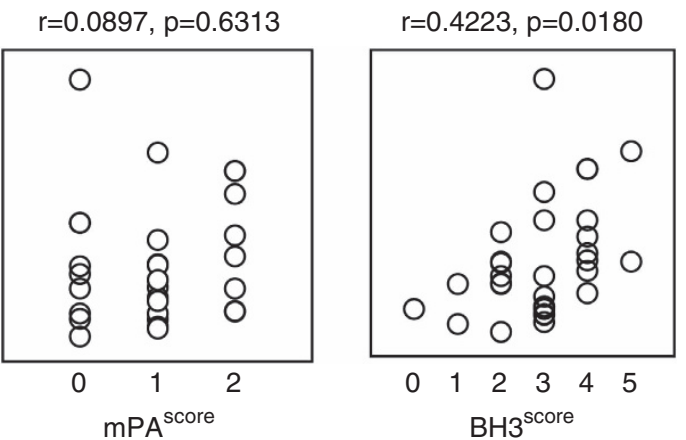

CPHSRA

$\mathrm{BH}^{\text {score }}<3$ vs. $\mathrm{BH}^{\text {score }}=3: \mathrm{p}=0.6343$

$\mathrm{BH}^{\text {score }}>3$ vs. $\mathrm{BH}^{\text {score }}=3: \mathrm{p}=0.2555$

$\mathrm{BH}^{\text {score }}>3$ vs. $\mathrm{BH}^{\text {score }}<3: \mathrm{p}=0.0132$

Figure 5 Overall survival versus subgroup of BCL-2 protein family. (a) Estimation of correlation (Pearson's test) between OS and the number of highly expressed antiapoptotic proteins in GBM (anti-apoptotic score $\left(A A^{\text {score }}\right)$ ), the number of highly expressed multi-domain pro-apoptotic proteins in GBM (multi-domain pro-apoptotic score $\left.\left(\mathrm{mPA}^{\text {score }}\right)\right)$, or the number of highly expressed BH3-only proteins in GBM (BH3 score (BH3 $\left.{ }^{\text {score }}\right)$ ). For the calculation of each score, 1 point was attributed when a member of the BCL-2 protein family harbored a high expression level (higher than the median), 0 point was attributed when the member of BCL-2 protein family harbored a low expression level (lower or equal to the median). (b) Kaplan-Meier curves illustrate the significant difference in OS between patients with tumors harboring more than three highly expressed $\mathrm{BH}$-only proteins $\left(\mathrm{BH}^{\text {score }}>3\right.$ ), and patients with tumors having less than three highly expressed $\mathrm{BH}$-only proteins.

another member of the BCL-2 protein family has been already suggested by the existence of a reciprocal protection of $\mathrm{Mcl}-1$ and Bim from ubiquitin-proteasome degradation. ${ }^{26}$

Taking into account the presence of p53 mutation in GBM, our study enters the debate on the influence of mutated p53 mutation on the expression of p53-regulated genes and as such on the expression of the proteins encoded by these genes. Our data suggest that the presence of mutated p53 correlates only with the expression level of Bak. This correlation is surprising as Bak is not a conventional p53regulated gene such as bax, puma or noxa for example. ${ }^{27,28}$

Finally, the description of the correlations between the expression levels of Bak and Bim and the methylation status of the MGMT gene suggests that these two pro-apoptotic proteins could have a crucial role into the temozolomide/ irradiation-induced apoptosis. In other terms, these data suggest that Bak and Bim are two crucial actors in the apoptosis induced by the temozolomide/irradiation treatment in cells harboring a MGMT methylation.

We also noted that 5/8 GBM tumors harboring the MGMT methylation highly co-expressed Bak and Bim proteins. Indeed, the determination of the expression of Bak and Bim as a means to further classify GBM populations harboring a methylated MGMT gene would be important in identifying among the methylated MGMT-patients those who are better responders to the irradiation-temozolomide treatment. Clearly, to be supported, this point requires a study with a bigger cohort of patients.

Taking into account the presence of p53 mutation in GBM, our study enters the debate on the influence of mutated p53 mutation on the expression of p53-regulated genes and as such on the expression of the proteins encoded by these genes. Our data suggest that the presence of mutated p53 correlates only with the expression level of Bak. This correlation is surprising as Bak is not a conventional p53regulated gene such as bax, puma or noxa for example. ${ }^{29-31}$

\section{Materials and methods}

Patient characteristics. OS was measured from the date of surgical resection to death. All patients included in this study had similar management and similar treatment (total resection, radiotherapy plus temozolamide). Patient material as well as records (diagnosis, age, sex, date of death and Karnofsky Performance Score (KPS)) was used with confidentiality according to French laws and recommendations of the French National Committee of Ethics.

Statistical analysis. Significance of correlations was determined using the Pearson's test. Survival curves were plotted according to Kaplan-Meier method and compared by the Cox proportional hazards survival regression analysis (CPHSRA) (such as indicated in the corresponding figures). 
a
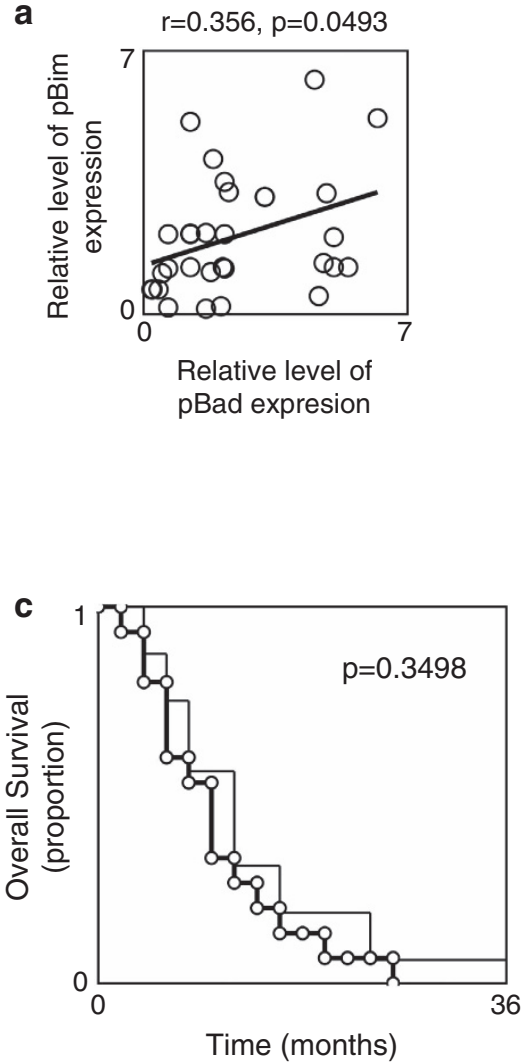

- Low pBim ${ }^{\mathrm{S} 69}, \mathrm{n}=16, \mathrm{~m}=12.4$ months

- High $\mathrm{pBim}^{\mathrm{S} 69}, \mathrm{n}=15, \mathrm{~m}=10.5$ months

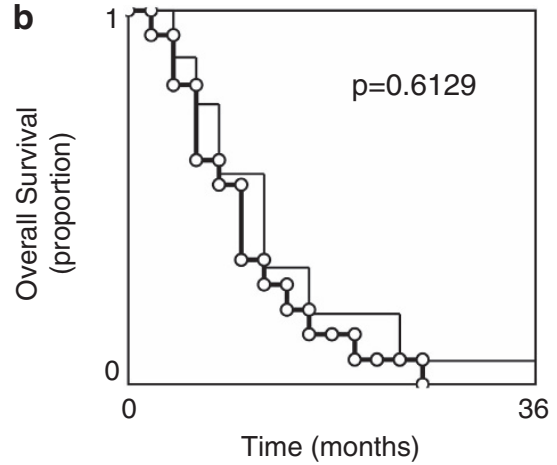

Low $\mathrm{pBad}^{\mathrm{S} 136}, \mathrm{n}=16, \mathrm{~m}=12.4$ months

$\multimap-$ High pBad $^{\mathrm{S} 136}, \mathrm{n}=15, \mathrm{~m}=10.5$ months

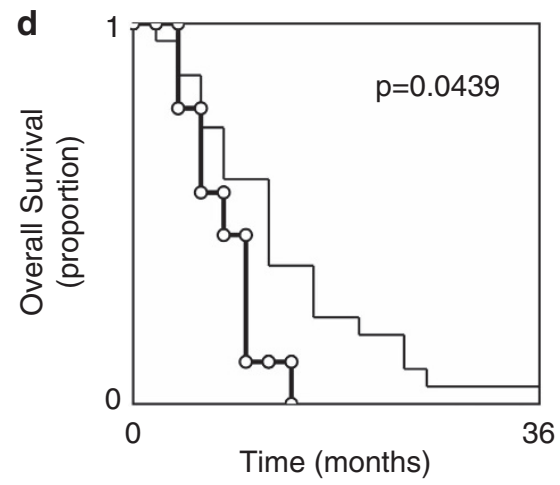

- rest, $\mathrm{n}=22, \mathrm{~m}=12.4$ month
- - High $\mathrm{pBad}^{\mathrm{s} 136} / \mathrm{pBim}^{\mathrm{s} 69}$,
$\mathrm{n}=9, \mathrm{~m}=9.4$ months

Figure 6 Overall survival versus Bad and/or Bim phosphorylation. (a) Estimation of correlation (Pearson's test) between the level of pBad ${ }^{\mathrm{S136}}$ and $\mathrm{pBim}{ }^{\mathrm{S69}}$. (b) KaplanMeier curves illustrate the absence of significant difference in OS between patients with tumors harboring low level of $\mathrm{pBad}{ }^{\mathrm{S} 136}$ and patients with high level of $\mathrm{pBad}{ }^{\mathrm{S136}}$. (c) Kaplan-Meier curves illustrate the absence of significant difference in OS between patients with tumors harboring low level of $p B i m^{569}$ and patients with high level of $\mathrm{pBim}^{\mathrm{S} 69}$. (d) Kaplan-Meier curves illustrate the significant difference in OS between patients with tumors harboring high levels of $\mathrm{pBim}^{\mathrm{S69}}$ and $\mathrm{pBad}{ }^{\mathrm{S} 136}$ and patients devoid of this double hallmark.

TP53 mutation analysis. To amplify TP53 exons 5-8, PCR was performed in a volume of $10 \mu \mathrm{l}$ containing $10 \mathrm{ng}$ DNA, $50 \mathrm{mmol} / \mathrm{l} \mathrm{KCl}, 10 \mathrm{mmol} / \mathrm{l}$ Tris- $\mathrm{HCl}$, $200 \mathrm{mmol} / \mathrm{l}$ each dNTP, $0.1 \%$ gelatin, $20 \mathrm{pmol}$ of each primer, $1-2 \mathrm{mmol} / \mathrm{I} \mathrm{MgCl}$ and $0.025 \mathrm{U}$ Taq polymerase. The primer sequences were exon 5a $5^{\prime}$-TCAA CTCTGTCTCCTTCCTC- $3^{\prime}$ and $5^{\prime}$-CTGTGACTGCTTGTAGATGG- $3^{\prime}$; exon 5b $5^{\prime}$-GTGGGTTGATTCCACACCCC-3' and $5^{\prime}$-AACCAGCCCTGTCGTCTCTC-3'; exon $65^{\prime}$-AGGCCTCTGATTCCTCACTG-3' $3^{\prime}$ and $5^{\prime}$-AGAGACCCCAGTTGCAA ACC-3'; exon 7 5'-GGCCTCATCTTGGGCCTGTG-3' and $5^{\prime}$-GTGTGCAGGGTG GCAAGTGG-3'; exon $85^{\prime}$-AATGGGACAGGTAGGACCTG-3' and 5'-ACCGCTTC TTGTCCTGCTTG- $3^{\prime}$. The PCR conditions were initially $94^{\circ} \mathrm{C}$ for $3 \mathrm{~min}$ followed by 30 cycles at $94^{\circ} \mathrm{C} / 30 \mathrm{~s}, 57^{\circ} \mathrm{C} / 40 \mathrm{~s}$, and extension at $72^{\circ} \mathrm{C}$ for $40 \mathrm{~s}$. A final extension step at $72{ }^{\circ} \mathrm{C}$ for $10 \mathrm{~min}$ was added. Single-strand conformation polymorphism analysis was performed on a sequencing apparatus using 8 and $14 \%$ acrylamide gels and electrophoresis at $3-6 \mathrm{~W}$ for $15 \mathrm{~h}$ followed by silver staining of the gels. Aberrantly migrating bands were excised and DNA was extracted. After reamplification with the same set of primers the PCR products were sequenced on a semi-automated sequencer (ABI 3100, Applied Biosystems) using a Taq cycle sequencing kit (Applied Biosystems, Villebon Sur Yvette, France). Each amplicon was sequenced bidirectionally.

MGMT methylation status. DNA was extracted using the QiaAmp DNA mini Kit (Qiagen, Courtaboeuf, France). Then a bisulfite conversion is performed using an EZ DNA methylation Gold kit (Zyma Research-Proteigene, St Marcel, France). MSP were done as previously described. ${ }^{32}$
Protein extraction, ELISA and western blot analysis. After surgical resection, the tumor samples were immediately frozen in liquid nitrogen and stored at $-80^{\circ} \mathrm{C}$. Protein extract was performed using vol/vol RIPA buffer (PBS containing $1 \% \mathrm{NP}-40,0.5 \%$ Na-deoxycholate, $0.1 \%$ sodium dodecyl sulfate (SDS), $1 \mathrm{~nm} \mathrm{Na-vanadate,} \mathrm{and} \mathrm{complete} \mathrm{inhibitor} \mathrm{cocktail).} \mathrm{In} \mathrm{brief,} \mathrm{proteins}(50 \mathrm{mg})$ were size fractionated by sodium dodecyl sulfate-polyacrylamide gel electrophoresis. Proteins were transferred onto nitrocellulose or PVDF membrane. Saturation and blotting were realized by using SNAP i.d Protein Detection System (Millipore, Molsheim, France) and the following antibodies (Actin no. MAB1501R, Millipore; Bad no. 610391, BD Bioscience, Le Pont de Claix, France; Bid no. AF860, R\&D system, Lille, France; Hrk no. sc-6973, Santa-Cruz/Tebu-Bio, Le Perray en Yvelines, France; Noxa no. sc-11718, Santa-Cruz/Tebu-Bio; Bax no. A3533, Dako, Trappes, France; Bcl-w no. 197209, Calbiochem/Millipore, Molsheim, France; Bak no. sc-832, Santa-Cruz/Tebu-Bio; Bcl-xl no. sc-7195, Santa-Cruz/Tebu-Bio; Bcl-2 no. sc-7382, Santa-Cruz/Tebu-Bio; and Puma no. P4743, Sigma, St Quentin Fallavier, France; Bok no. sc-7382, Santa-Cruz/Tebu-Bio; Bmf no. sc-20182, Santa-Cruz/ Tebu-Bio; Mcl-1 no. sc-74437, Santa-Cruz/Tebu-Bio; Bik no. sc-101214, Santa-Cruz/ Tebu-Bio). The detection of proteins was performed using ECL (GE-Amersham Biosciences, Velizy, France) and/or SuperSignal west femto Maximum Sensitivity (Pierce/Thermo Scientific, Courtaboeuf, France) chemilumenscence reagents. Bands were quantified using Quantity One quantification software (Bio-Rad, Marnes-laCoquette, France). Value was normalized using actin as internal standard.

The expression of Bad, Bim, pBad and pBim was estimated by ELISAs according to the manufacturer's instructions (PathScan Total Bad Sandwich 
a

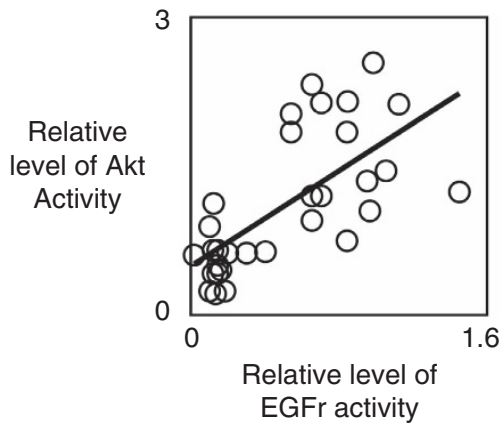

b

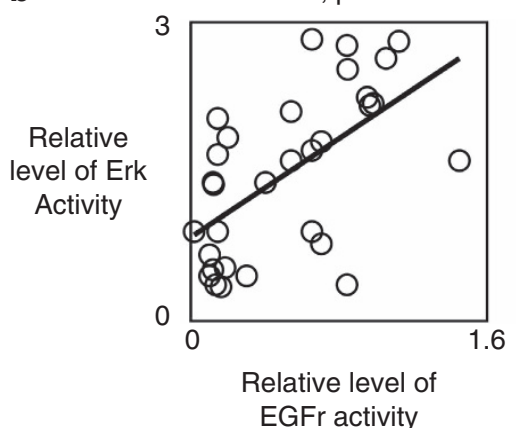

$r=0.520, p=0.0027$

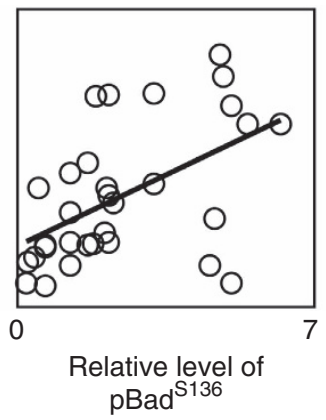

$r=0.321, p=0.0783$

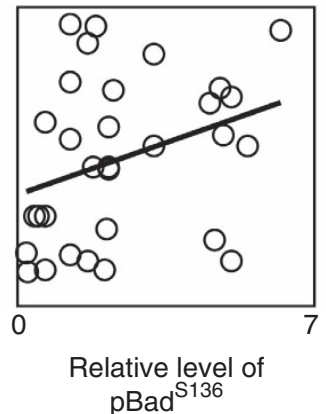

$r=0.334, p=0.0581$

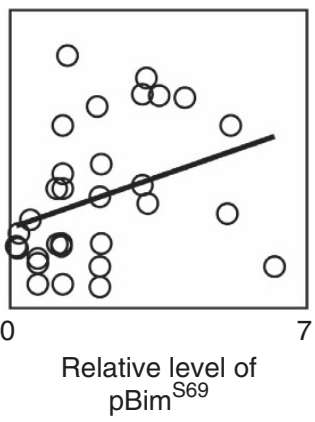

$r=0.694, p<0.0001$

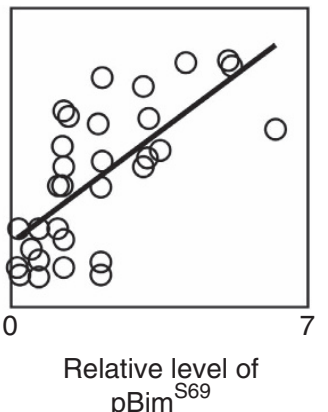

Figure 7 Presence of $\mathrm{pBad}^{\mathrm{S136}}$ and $\mathrm{pBim}{ }^{\mathrm{S69}}$ in GBM and kinase activities. (a) Graphs and Pearson's test analyze the presence of correlation between the levels of Akt activity and the level of EGFr activity, or the level of $\mathrm{pBad}^{\mathrm{S} 136}$ or the level of $\mathrm{pBim}{ }^{\mathrm{S69}}$. (b) Graphs and Pearson's test analyze the presence of correlation between the levels of Erk activity and the level of EGFr activity, or the level of $\mathrm{pBad}^{\mathrm{S} 136}$ or the level of $\mathrm{pBim}{ }^{\mathrm{s} 69}$.

ELISA Kit (Cell Signaling/Ozyme, St Quentin en Yvelines, France) and Human BIM ELISA Kit (Tebu-Bio, Le Perray en Yvelines, France)) and the $\mathrm{pBad}^{\text {S136 }}$ and $\mathrm{pBim}^{\mathrm{S69}}$ antibodies (Cell Signaling/Ozyme, France).

\section{Conflict of Interest}

The authors declare no conflict of interest.

Acknowledgements. This work was supported by a grant from the 'Association pour la Recherche contre le Cancer' (ARC) and the 'Ligue contre la Cancer Grand Ouest'. We thank Dr Lisa Oliver for critical reading of the manuscript and fruitful discussions.

1. Van Meir E, Hadjipanayis C, Norden A, Shu H, Wen P, Olson J. Exciting new advances in neurooncology: the avenue to a cure for malignant glioma. CA Cancer J Clin 2010; 60: 166-193.

2. Stupp R, Mason W, van den Bent M, Weller M, Fisher B, Taphoorn M et al. Radiotherapy plus concomitant and adjuvant temozolomide for glioblastoma. N Engl J Med 2005; 352 987-996.

3. Chakravarti A, Erkkinen M, Nestler U, Stupp R, Mehta M, Aldape K et al. Temozolomidemediated radiation enhancement in glioblastoma: a report on underlying mechanisms. Clin Cancer Res 2006; 12: 4738-4746.

4. Bucci B, Misiti S, Cannizzaro A, Marchese R, Raza G, Miceli R et al. Fractionated ionizing radiation exposure induces apoptosis through caspase-3 activation and reactive oxygen species generation. Anticancer Res 2006; 26: 4549-4557.

5. Günther W, Pawlak E, Damasceno R, Arnold H, Terzis A. Temozolomide induces apoptosis and senescence in glioma cells cultured as multicellular spheroids. Br J Cancer 2007; 88: 463-469.

6. Danial N, Korsmeyer S. Cell death: critical control points. Cell 2004; 116: 205-219.

7. Lalier L, Cartron P, Juin P, Nedelkina S, Manon S, Bechinger B et al. Bax activation and mitochondrial insertion during apoptosis. Apoptosis 2007; 12: 887-896.

8. Reed J. Proapoptotic multidomain Bcl-2/Bax-family proteins: mechanisms, physiological roles, and therapeutic opportunities. Cell Death Differ 2006; 13: 1378-1386.

9. Shamas-Din A, Brahmbhatt H, Leber B, Andrews D. BH3-only proteins: orchestrators of apoptosis. Biochem Biophys Acta 2011; 1813: 508-520.
10. Certo M, Del Gaizo Moore V, Nishino M, Wei G, Korsmeyer S, Armstrong S et al. Mitochondria primed by death signals determine cellular addiction to antiapoptotic BCL-2 family members. Cancer Cell 2006; 9: 351-365.

11. Daneshbod Y, Amirghofran Z, Tabei S. Bcl-2 expression in acute myelogenous leukemia: the relation to myeloid antigen expression and response to therapy in Iranian patients. Neoplasma 2005; 52: 109-114.

12. Buchholz T, Davis D, McConkey D, Symmans W, Valero V, Jhingran A et al. Chemotherapy-induced apoptosis and Bcl-2 levels correlate with breast cancer response to chemotherapy. Cancer J 2003; 9: 33-41.

13. Coutinho-Camillo C, Lourenço S, Nishimoto I, Kowalski L, Soares F. Expression of Bcl-2 family proteins and association with clinicopathological characteristics of oral squamous cell carcinoma. Histopathology 2010; 57: 304-316.

14. Elkholi R, Floros K, Chipuk J. The role of BH3-Only proteins in tumor cell development, signaling, and treatment. Genes Cancer 2011; 2: 523-537.

15. Puthalakath $\mathrm{H}$, Strasser $\mathrm{A}$. Keeping killers on a tight leash: transcriptional and posttranslational control of the pro-apoptotic activity of BH3-only proteins. Cell Death Differ 2002; 9: 505-512.

16. Basu A, DuBois G, Haldar S. Posttranslational modifications of Bcl2 family members-a potential therapeutic target for human malignancy. Front Biosci 2006; 11 : $1508-1521$.

17. del Peso L, González-García M, Page C, Herrera R, Nuñez G. Interleukin-3-induced phosphorylation of BAD through the protein kinase Akt. Science 1997; 278: 687-689.

18. Ley R, Balmanno K, Hadfield K, Weston C, Cook S. Activation of the ERK $1 / 2$ signaling pathway promotes phosphorylation and proteasome-dependent degradation of the BH3-only protein, Bim. J Biol Chem 2003; 278: 18811-18816.

19. Longley D, Johnston P. Molecular mechanisms of drug resistance. J Pathol 2005; 205: 275-292.

20. Dittadi R, Catozzi L, Gion M, Brazzale A, Capitanio G, Gelli M et al. Comparison between western blotting, immunohistochemical and ELISA assay for p185neu quantitation in breast cancer specimens. Anticacer res 1993; 13: 1821-1824.

21. Martin S, Toquet $\mathrm{C}$, Oliver L, Cartron $\mathrm{P}$, Perrin $\mathrm{P}$, Meflah $\mathrm{K}$ et al. Expression of bcl-2, bax and bcl-xl in human gliomas: a re-appraisal. J Neurooncol 2001; 52: 129-139.

22. Dai D, Wang Y, Liu M, Martinka M, Li G. Bim expression is reduced in human cutaneous melanomas. J Invest Dermatol 2008; 128: 403-407.

23. Sinicrope F, Rego R, Foster N, Thibodeau S, Alberts S, Windschitl H et al. Proapoptotic Bad and Bid protein expression predict survival in stages II and III colon cancers. Clin Cancer Res 2008; 14: 4128-4133. 
24. Sinicrope F, Rego R, Okumura K, Foster N, O'Connell M, Sargent D et al. Prognostic impact of bim, puma, and noxa expression in human colon carcinomas. Clin Cancer Res 2008; $14: 5810-5818$.

25. Hervouet $E$, Vallette $F$, Cartron $P$. Impact of the DNA methyltransferases expression on the methylation status of apoptosis-associated genes in glioblastoma multiforme. Cell Death Dis 2010; 1: e8.

26. Wuillème-Toumi S, Trichet V, Gomez-Bougie P, Gratas C, Bataille R, Amiot M. Reciprocal protection of Mcl-1 and Bim from ubiquitin-proteasome degradation. Biochem Biophys Res Commun 2007; 361: 865-869.

27. Villunger A, Michalak E, Coultas L, Müllauer F, Böck G, Ausserlechner M et al. p53- and drug-induced apoptotic responses mediated by $\mathrm{BH} 3$-only proteins puma and noxa. Science 2003; 302: 1036-1038.

28. Miyashita T, Reed J. Tumor suppressor p53 is a direct transcriptional activator of the human bax gene. Cell 1999; 80: 293-299.

29. Jha $P$, Suri $V$, Jain $A$, Sharma $M$, Pathak $P$, Jha $P$ et al. O6-methylguanine DNA methyltransferase gene promoter methylation status in gliomas and its correlation with other molecular alterations: first Indian report with review of challenges for use in customized treatment. Neurosurgery 2010; 67: 1681-1691.
30. Weller M, Felsberg J, Hartmann C, Berger H, Steinbach J, Schramm J et al. Molecular predictors of progression-free and overall survival in patients with newly diagnosed glioblastoma: a prospective translational study of the German Glioma Network. J Clin Oncol 2009; 27: 5743-5750

31. Groenendijk F, Taal W, Dubbink H, Haarloo C, Kouwenhoven M, van den Bent M et al. MGMT promoter hypermethylation is a frequent, early, and consistent event in astrocytoma progression, and not correlated with TP53 mutation. J Neurooncol 2011; 101: 405-417.

32. Esteller M, Garcia-Foncillas J, Andion E, Goodman SN, Hidalgo OF, Vanaclocha V et al. Inactivation of the DNA-repair gene MGMT and the clinical response of gliomas to alkylating agents. N Engl J Med 2000; 343: 1350-1354.

Cell Death and Disease is an open-access journal published by Nature Publishing Group. This work is licensed under the Creative Commons Attribution-NonCommercialShare Alike 3.0 Unported License. To view a copy of this license, visit http://creativecommons.org/licenses/by-nc-sa/3.0/ 